\title{
Cosmological evolution of radio galaxies
}

\section{Yang Wang ${ }^{1}$}

University of Southampton

School of Physics \& Astronomy, University of Southampton, SO17 1BJ, UK

E-mail: wangyang@soton.ac.uk

\section{Christian Kaiser}

\section{University of Southampton}

School of Physics \& Astronomy, University of Southampton, SO17 1BJ, UK

E-mail: crk@phys.soton.ac.uk

By combining a model for the evolution of the radio luminosity of an individual source with the radio luminosity function, we perform a multi-dimensional Monte-Carlo simulation to investigate the cosmological evolution of the Fanaroff-Riley Class II radio galaxy population by generating large artificial samples. The properties of FRII sources

are required to evolve with redshift in the artificial samples to fit the observations. Either the maximum jet age or the maximum density of the jet environment or both evolve with redshift. We also study the distribution of FRII source properties as a function of redshift. From currently available data we can not constrain the shape of the distribution of environment density or age, but jet power is found to follow a power-law distribution with an exponent of -2 . This powerlaw slope does not change with redshift out to $\mathrm{z}=0.6$. We also find the distribution of the pressure in the lobes of FRII sources to evolve with redshift up to $\mathrm{z} \sim 1.2$

VII Microquasar Workshop: Microquasars and Beyond

Foca, Izmir, Turkey

September $1^{\text {st }}-5^{\text {th }} 2008$

\footnotetext{
$1 \quad$ Poster Presenter
} 


\section{Introduction}

The formation and evolution of massive galaxies are important components in understanding how the universe evolved. It is not clear why AGN formation takes place at the centres of some galaxies, but it is believed that this occurs as part of the formation of all massive galaxies and jet activity is often associated with AGN. Some of the most distant and powerful active galaxies have been observed in the radio band and radio astronomy has been closely connected with cosmology ever since.

\section{Radio luminosity function}

In recent years, various samples of radio sources observed at low radio frequencies have been published like 3CRR, 6CE and 7CRS, which are all complete down to their fluxlimits. The radio luminosity function (RLF) generated by these complete sample has been developed as a tool to describe the space densities of radio sources. Willott et al. (2001) generated an RLF at $151 \mathrm{MHz}$ which shows a peak at around $z=2$. The shape of the RLF is shown in Fig.1

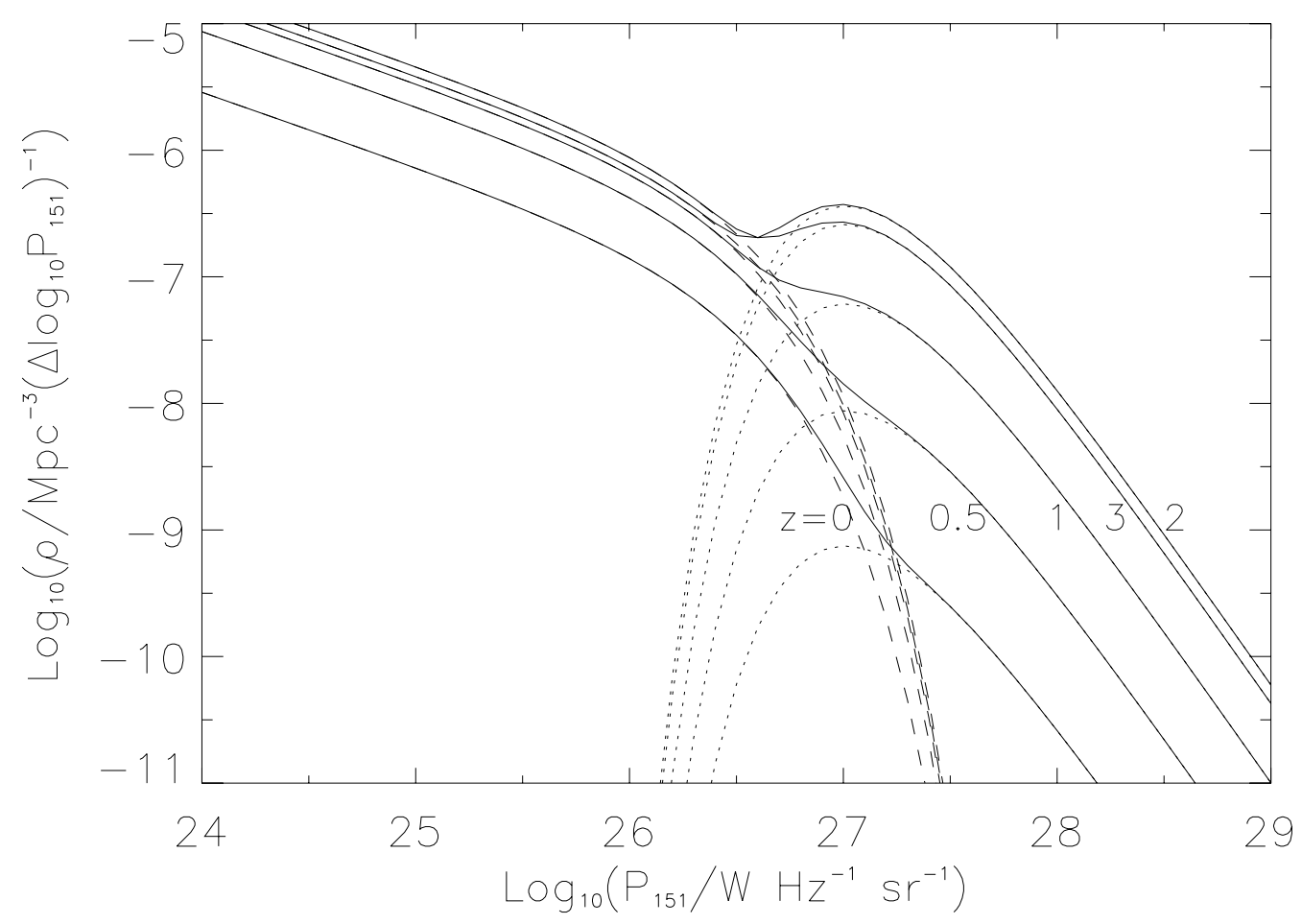

Fig.1 Radio luminosity function at $151 \mathrm{MHz}$, from Willott et al. 2001. The solid lines show the total function, the dashed lines show the low-luminosity population and the dotted lines indicate the high-luminosity population. 


\section{FRII models}

Many analytical models for extragalactic classical double radio sources have been published which characterize radio sources in terms of their dynamics and radio luminosity evolution. Kaiser, Dennett-Thorpe \& Alexander (1997, hereafter KDA) takes into account the energy losses of electrons due to the adiabatic expansion of the lobes, synchrotron radiation and inverse Compton scattering of cosmic microwave background photons off the electrons. The KDA model tracks the luminosity evolution of a radio source through the power-linear size (P-D) diagram, which is shown in Fig.2. Manolakou \& Kirk (2002, hereafter MK) added the effect of energy losses within the head region at the end of the jet (identified with the radio hotspots in observations) to the model of KDA. Blundell, Rawlings \& Willott\{1999, hereafter BRW\} assume further that the hotspot has a fixed physical size and that it takes electrons between 1 and 100,000 years to escape from the hotspot into the lobe. After the electrons escape into the lobe, they preserve their double power-law energy distribution, but the breaks in the power-law evolve with time. These models give similar simulation result. We just concentrate KDA model as it is mathematically simplest.

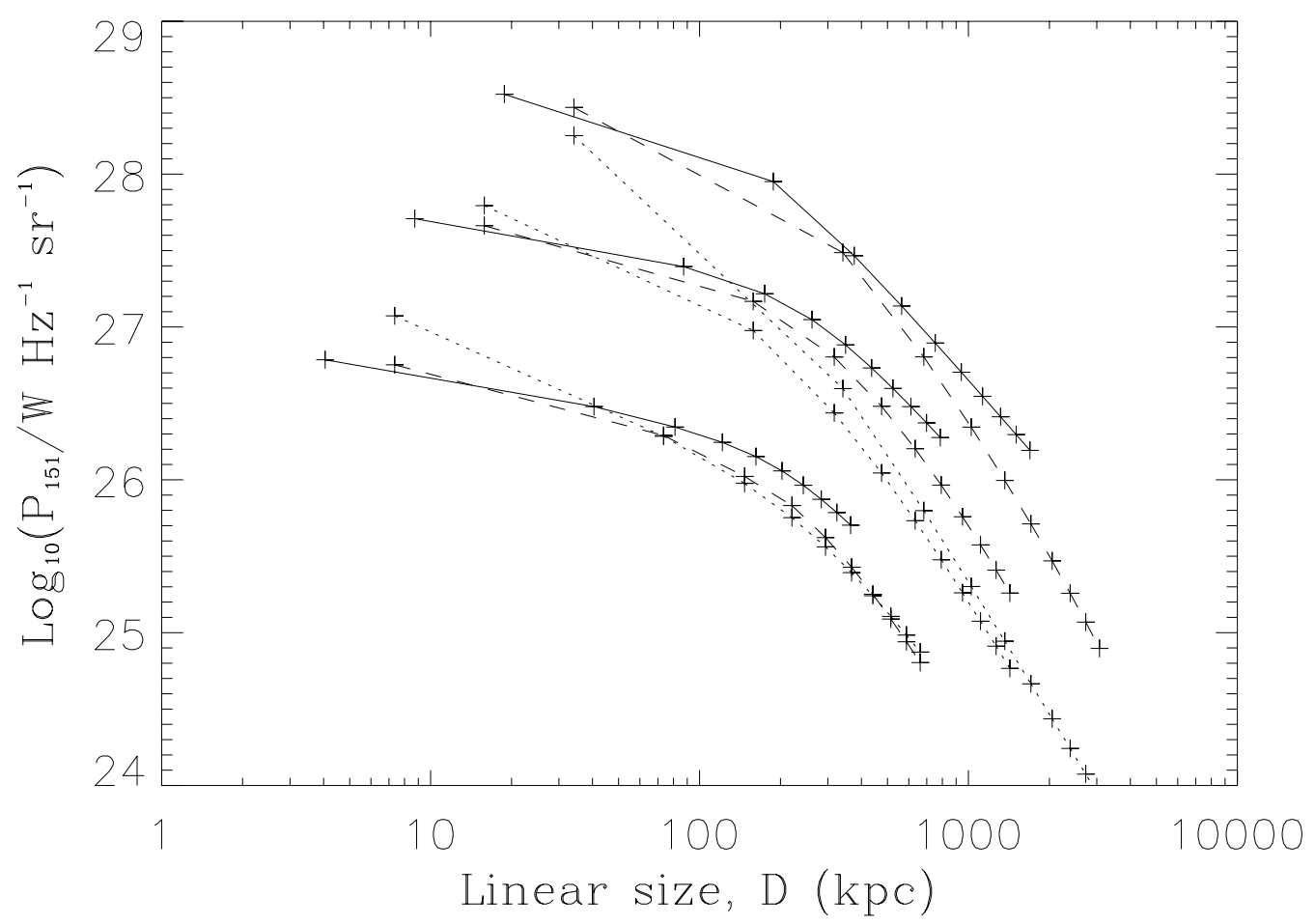

Fig. 2 Evolutionary track for three sources with different models. The upper curves are for $\log (Q)=40$ and $z=1.5$, the centre curves are for $\log (Q)=39$ and $z=0.5$, and the lower curves are for $\log (Q)=38$ and $z=0.2$. Each of the solid, dashed and dotted curves refer to the tracks given by KDA, MK and MK models respectively. The pluses on the curves are time markers denoting source lifetimes of $1,10,20, \ldots 90 \mathrm{Myr}$. 


\section{Simulation result}

Using the KDA model, we generate artificial samples of radio galaxies with the same selection criteria as the observed samples. We can then compare these to the observational results in the radio luminosity, size, redshift data cube. For this we take the radio luminosity function as the 'birth function' of radio galaxies for a given redshift. The model parameters defining the size and luminosity of a source like jet power, source lifetime and density of the source environment, are assigned through a Monte Carlo simulation.

Comparing our artificial samples to observations, we find that some of the model parameters require some evolution with redshift. Both the lifetime of the jet flows, $t_{0}$, and the density of the source environment need to change with $\mathrm{z}$. The latter is modeled as a power-law where $\rho_{0}$ is the density with a distance $a_{0}$ from the centre. If $\mathrm{N}$ is a random number between 0 and 1 , then the source age, $t_{0}$, and $\rho_{0}$ are given by: $t_{0}=r_{N} \times 3 \times 10^{8} \mathrm{yr} /(1+z)^{\alpha}$ $\Lambda=\frac{7}{16} \rho_{0} a_{0}{ }^{2}=r_{N} \times 3 \times 10^{19} \mathrm{kgm}^{-1} \times(1+z)^{\beta}$

The distribution of source in our artificial sample as shown by contours in Fig. 3 depend on the values of $\alpha$ and $\beta$. Here we use $\alpha=2.0$ and $\beta=1.2$
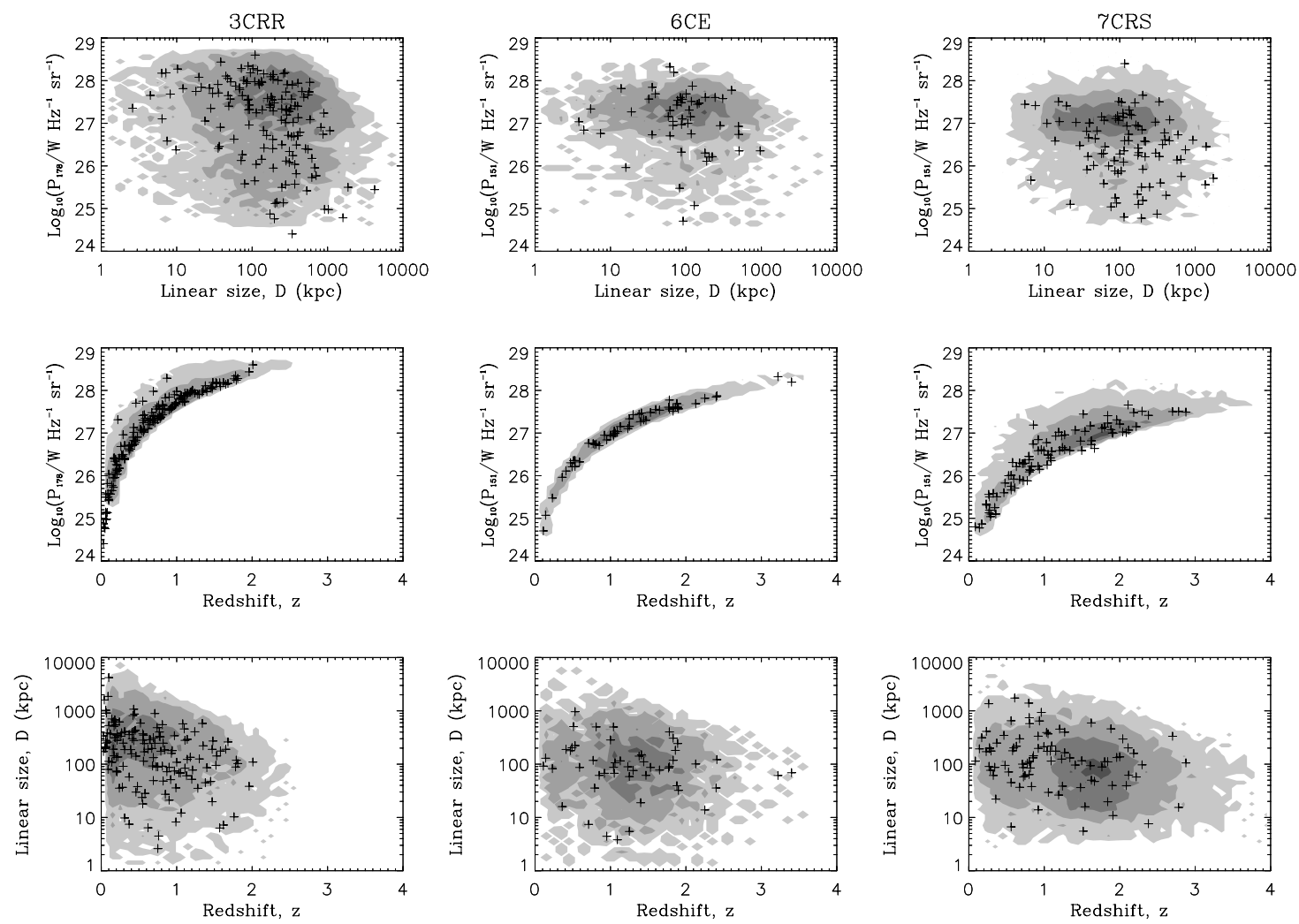

Fig. 3 Simulation result using the KDA model. The filled contours show the source distribution of our artificial sample, while pluses show sources in the observed samples indicated at the top of each panel. 


\section{References}

[1] Blundell K.M., Rawlings S., Willott C.J., 1999, ApJ, 117, 677

[2] Kaiser C.R., Alexander P., 1997, MNRAS, 286, 215

[3] Kaiser C.R., Dennett-Thorpe J., Alexander P., 1997, MNRAS, 292, 723

[4] Manolakou K., Kirk J.G. 2002, A\&A, 391, 127.

[5] Willott C.J., Rawlings S., Blundell K.M., Lacy M., Eales S.A., 2001, MNRAS, 322, 536

[6] Wang Y., Kaiser C.R., 2008, MNRAS, 388, 677 